\title{
Soiling and other optical losses in solar-tracking PV plants in Navarra
}

\author{
Miguel García ${ }^{1 *}$, Luis Marroyo ${ }^{1}$, Eduardo Lorenzo ${ }^{2}$ and Miguel Pérez ${ }^{3}$ \\ 1 Dpto. Ingeniería Eléctrica y Electrónica, Universidad Pública de Navarra, Campus Arrosadia, 31006 Pamplona, Spain \\ 2 Instituto de Energía Solar, ETSI Telecomunicación, Ciudad Universitaria, s/n, 28040, Madrid, Spain \\ 3 Acciona Solar, Avda. de la Ciudad de la Innovación, 331621 Sarriguren, Navarra, Spain
}

\begin{abstract}
Field data of soiling energy losses on PV plants are scarce. Furthermore, since dirt type and accumulation vary with the location characteristics (climate, surroundings, etc.), the available data on optical losses are, necessarily, site dependent. This paper presents field measurements of dirt energy losses (dust) and irradiance incidence angle losses along 2005 on a solar-tracking PV plant located south of Navarre (Spain). The paper proposes a method to calculate these losses based on the difference between irradiance measured by calibrated cells on several trackers of the PV plant and irradiance calculated from measurements by two pyranometers (one of them incorporating a shadow ring) regularly cleaned. The equivalent optical energy losses of an installation incorporating fixed horizontal modules at the same location have been calculated as well. The effect of dirt on both types of installations will accordingly be compared. Copyright (C) 2010 John Wiley \& Sons, Ltd.

KEYWORDS

soiling, optical losses, angle of incidence, large grid-connected PV plants, PV tracking systems

${ }^{*}$ Correspondence

Miguel García, Edificio Los Pinos, Dpto. Ingeniería Eléctrica y Electrónica, Universidad Pública de Navarra, Campus Arrosadia, 31006 Pamplona, Spain.

E-mail: miguel.garcia@unavarra.es
\end{abstract}

\section{INTRODUCTION}

The radiation received by the cells inside a PV module is significantly lower than the radiation arriving to the module surface The main causes of this energy loss are dirt accumulated on the surface (dust, pollution...) of the modules and reflection and absorption losses by the materials covering the cells. The reflection and adsorption losses depend on the radiance incidence angle [1-3], thus they are normally referred to as angle of incidence (AOI) losses. The AOI losses tend to increase as dirt on the surface augments [3,4], hence it is reasonable to study them concurrently. The term Optical losses encompass both types of energy losses. The value of the optical losses is essential to estimate and analyze the energy behavior of a $\mathrm{PV}$ installation.

Soiling energy loss field data are scarce in the literature. More to the point, since type and accumulation of dirt depend on the climate (rainfall, etc.), location surroundings and the position and inclination of the PV modules, available data on optical losses are, necessarily, site dependent [5-9]. The interest on studying dirt energy losses particular to different locations is patent.

In the literature, each field analysis treats soiling energy losses in a particular way. For instance, Hammond et al. in 1997 [5] recorded throughout a year the effect of dirt on the short-circuit current of several modules assembled into a two-axis PV tracker (Phoenix, Arizona). These data were compared with other identical modules placed on the same tracker but regularly cleaned. From the comparison of both datasets, it was concluded that rainfall rates over $5 \mathrm{~mm}$ reduced dirt energy losses (due solely to dust) approximately $0.5 \%$. Also, the maximum dirt energy losses registered during rain periods on tracking PV modules were $3 \%$. As a conclusion, the influence of the incidence angle on radiation energy losses due to dirt was confirmed - but not quantified.

Becker et al. [6] and Haeberling et al. [7] analyzed the reduction of the annual efficiency of fixed PV facilities with a $30^{\circ}$ inclination angle located near urban areas, thus 
close to pollution sources such as train stations or chimneys and biological pollution sources such as forests, farms, etc. The first of these studies reported that regular cleaning of the modules resulted on an energy ranging from 2 to $6 \%$. Haeberling et al. determined that, in 4 years, the generator power had decreased between 8 and $10 \%$ during the summer months due to pollution.

Kimber et al. [8] tried to simulate dirt energy losses in arid climates as a function of rainfall data and number of manual clean-ups. So, they suggested a linear model to represent the daily system efficiency reduction due to dirt (dust) between rainfalls. To adjust this model to a choice of locations, several datasets of the system efficiency during the dry season were used. The daily dirt energy loss rates range from 0.1 to $0.3 \%$. The model was also used [10] to estimate year dirt energy losses in the Los Angeles area (south of California). This analysis determined energy losses of about $5 \%$.

In spite of the fact that Hammond et al. analysis showed that there was a dependency between the irradiation incidence angle and dirt energy losses, none of the studies mentioned hereby took it into consideration. As a matter of fact, although year energy losses due to incidence angle are frequently estimated $[4,10]$, field measurements are scarce and the existing ones do not account for dirt energy losses [2].

This paper presents field measurements of dirt energy losses (dust) and irradiance incidence angle losses along 2005 on a PV plant located south of Navarre (Spain). The plant incorporates single vertical-axis trackers (azimuth tracking systems) tilted $45^{\circ}$ from the horizontal plane. The analysis methodology is based on the difference between the irradiance measured by three calibrated cells placed on separated trackers and the irradiance measured by two horizontal pyranometers (one of them incorporating a shadow ring) regularly cleaned. Likewise, the equivalent dirt and incidence angle losses of a fixed horizontal plant have been determined based on the measurements of three horizontal cells placed along the plant. The effect of dirt on both types of installations will accordingly be compared.

\section{LOCATION CHARACTERISTICS}

The plant "Montes del Cierzo" has a total power of $1.2 \mathrm{MWp}$ and integrates 400 single vertical-axis trackers (azimuth tracking) with $45^{\circ}$ tilted modules (Figure 1).

The plant is located in Tudela (North of Spain) in a rural area surrounded mainly by crops (cereal and some trees) and forest (conifers). Approximately $1 \mathrm{~km}$ away, there is a road with regular traffic flow (Figure 2).

Table I summarizes the main climate particularities of the area.

In this area, it rains mostly during spring and autumn. Because of the particularities of the area, the dirt accumulated on the modules is basically dust, although bird droppings are often found on the panels (Figure 3).

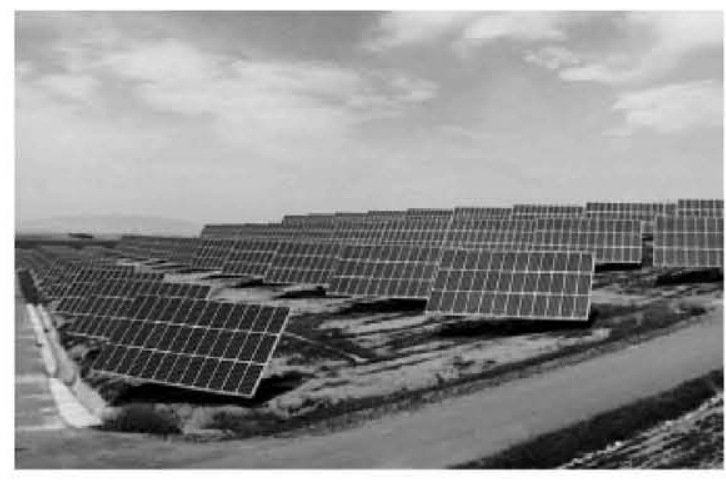

Figure 1. "Montes del Cierzo" PV plant.

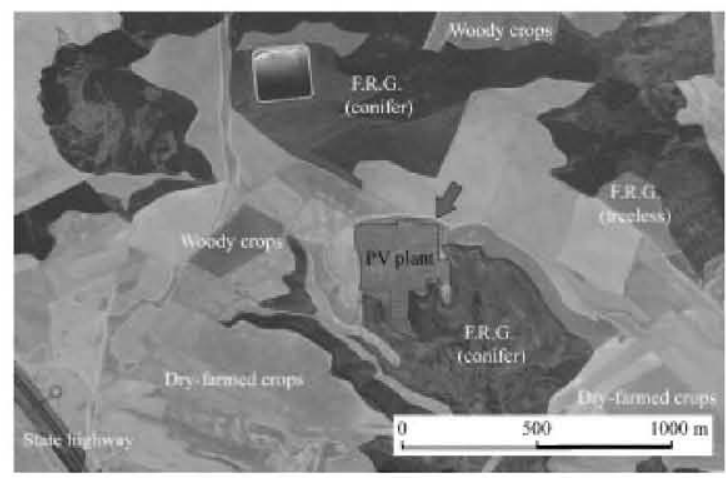

Figure 2. Satellite image of the PV plant and surroundings. The blue colored areas correspond to forestall rural ground (F.R.G.) composed of conifers; the green colored areas correspond to treeless forestall rural ground; the light brown colored areas indicate dry-farmed crop lands (cereals) and the dark brown colored ones indicate woody crop lands.

\section{METHODOLOGY}

There are six calibrated cells distributed along the plant, all of them with the same technology as the cells of the PV modules (Equivalent Technology Cells). The cells are placed on three different trackers. Three of these cells are placed on the same plane as the modules and the other three

Table I. Main climate data characteristic of the Tudela area (lat $\left.42.1^{\circ}\right)$

\begin{tabular}{lcr}
\hline & $\begin{array}{c}10 \text { year } \\
\text { average }\end{array}$ & 2005 \\
\hline Year horizontal irradiance $\left(\mathrm{kWh} / \mathrm{m}^{2}\right)$ & 1680 & 1619 \\
Year rainfall $(\mathrm{mm})$ & 370 & 291 \\
Spring rainfall $(\mathrm{mm})$ & 125 & 100 \\
Summer rainfall $(\mathrm{mm})$ & 75 & 30.9 \\
Autumm rainfall $(\mathrm{mm})$ & 100 & 128.4 \\
Winter rainfall $(\mathrm{mm})$ & 80 & 31.9 \\
Daily maximum rainfall $(\mathrm{mm})$ & 60 & 33.4 \\
Mean wind speed $(\mathrm{m} / \mathrm{s})$ & $4 \pm 2$ & 4.3 \\
\hline
\end{tabular}




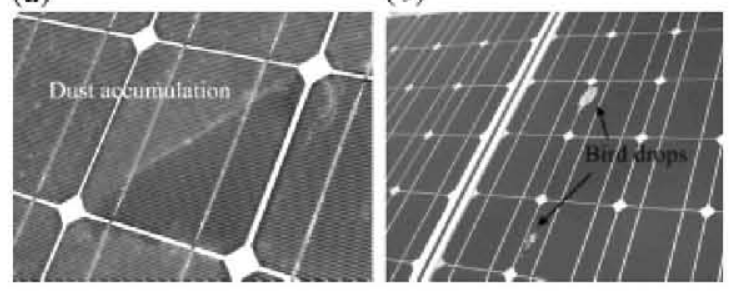

Figure 3. (a) Dust deposits on a PV module and (b) bird excrements on a PV module.

are on the horizontal plane (Figure 4). The irradiance incident on the cells is calculated through measurements of the short-circuit current and temperature. So, the temperature effect on the short-circuit current can be corrected according to a current temperature factor. Cell calibration uncertainty is approximately $\pm 2 \%$.

Since these cells are not cleaned up, the dirt deposited on the cell surface can be assumed to be the same as the dirt accumulated on the PV modules. It should be pointed out that, during the study, a particular effort has been made to

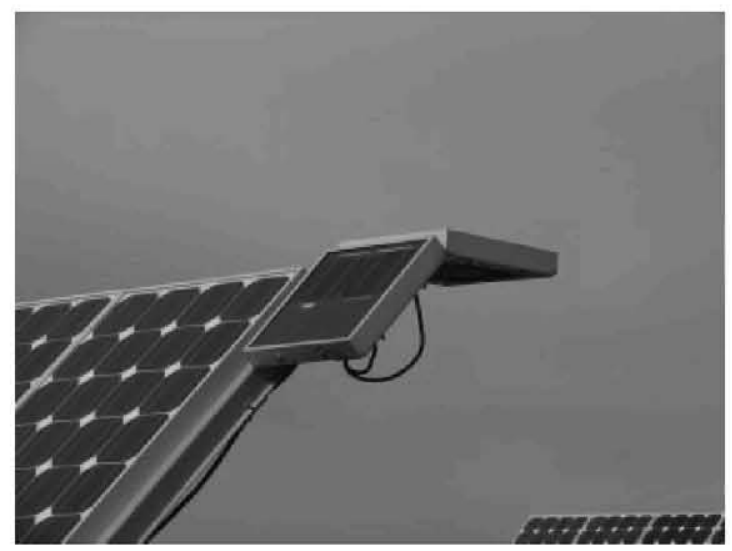

Figure 4. Keierence cells placed on one of trackers of the PV plant.

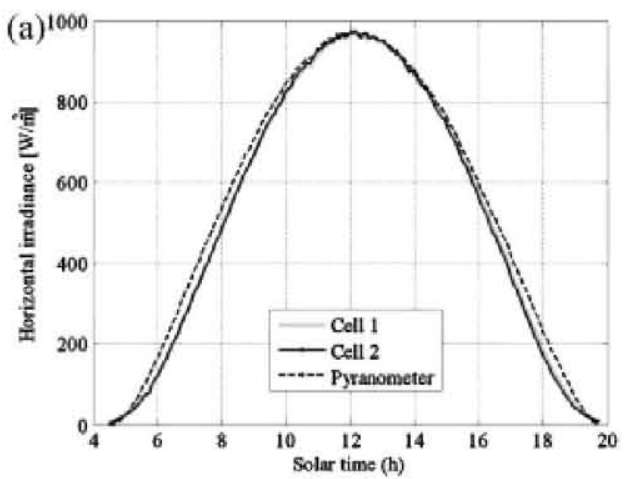

eliminate localized dirt, such as bird drops, on the modules. This type of dirt is only found on certain generators and depending on its size it can short-circuit bypass diodes in the modules. The scope of this study is to ascertain the influence of dirt that affects in a similar way all the generators, mainly dust.

The global and diffuse horizontal radiation is measured by means of 2 Kipp \& Zonen CM11 pyranometers, one of them incorporating a shadow ring. These pyranometers are regularly cleaned; hence the measurements can be considered not to be affected by dirt. Besides, the pyranometer cosine responses can be considered to be perfect [11]; that is to say, the incidence angle losses can be neglected (except for very high incidence angles). The expected daily accuracy of the pyranometer measurements is $\pm 3 \%$.

In order to reduce the influence of the uncertainty in the cells and pyranometer measurements, all the devices were previously assembled in horizontal position and adjusted so that the difference in measurements at midday of a summer clear day was in the range of $\pm 1 \%$ (on a summer day, near midday, the influence of the AOI is negligible). Figure 5.a shows the horizontal irradiance measured by the pyranometer and two of the calibrated cells during a clear day. Both the cells and the pyranometer were cleaned prior to start measuring. As shown in Figure 5.b, the difference between measurements is in the range of $\pm 1 \%$.

\subsection{Theoretical model}

Martin and Ruiz have proposed a model to describe the incidence angle losses as a function of the amount of dirt [3]. The model includes an expression for the angular loss factor of the solar radiation direct component, $\mathrm{FT}_{B}$ $\left(\theta_{S}\right)$, defined as the ratio between the transmittance at a certain incidence angle, $\theta_{S}$, and the transmittance at normal incidence:

$$
\mathrm{FT}_{B}\left(\theta_{S}\right)=1-\frac{\exp \left(-\frac{\cos \theta_{S}}{a_{r}}\right)-\exp \left(-\frac{1}{a_{r}}\right)}{1-\exp \left(-\frac{1}{a_{r}}\right)}
$$

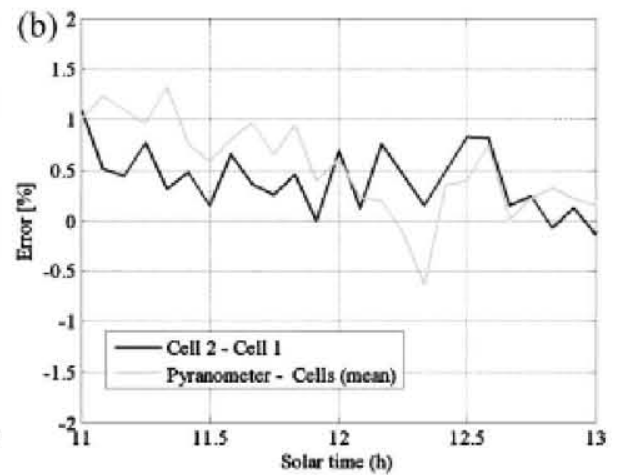

Figure 5. (a) Horizontal irradiance measured by the pyranometer and two of the calibrated cells during a clear day and (b) difference between measurements provided by the cells and the pyranometer near the midday. 
Table II. Typical values of $a_{r}$ as a function of dirt accumulated on the surface

\begin{tabular}{lcl}
\hline Dirt amount & $T_{\text {soiled }} / T_{\text {clean }}$ & $a_{r}$ \\
\hline Clean & 1 & 0.17 \\
Low & 0.98 & 0.2 \\
Medium & 0.97 & 0.21 \\
High & 0.92 & 0.27 \\
\hline
\end{tabular}

where $a_{r}$ is an adjustable parameter function of dirt accumulation on the surface. The amount of dirt on the surface can be characterized by the ratio between the normal incidence transmittance on a soiled surface and the normal incidence transmittance on a clean surface, $T_{\text {soiled }} / T_{\text {clean }}$. Table II includes typical values of $a_{r}$ as a function of the amount of dirt on the surface.

Equation (1) can be applied not only to the direct radiation, $B$, but also to the circumsolar component of the diffuse radiation, $D_{C}$. The same paper [3] includes formula to calculate the angular loss factor for the isotropic component of the diffuse radiation, $\mathrm{FT}_{D}$, and the angular losses factor for the reflected radiation, $\mathrm{FT}_{R}$. Besides, Hay et al. presented a model to obtain the isotropic component, $D_{I}$, and the circumsolar component, $D_{C}$, of the diffuse irradiance over a tilted surface based on the horizontal irradiance without the need to use any empirical parameters [12].

$$
\begin{gathered}
D=D_{I}+D_{C} \\
D_{I}=D(0)\left(1-k_{1}\right) \frac{1+\cos \beta}{2} \\
D_{C}=\frac{D(0) k_{1}}{\cos \theta_{Z S}} \max \left(0, \cos \theta_{S}\right)
\end{gathered}
$$

where

$$
k_{1}=\frac{B(0)}{B_{0}(0)}=\frac{B(0)}{B_{0} \epsilon_{0} \cos \theta_{\mathrm{ZS}}}
$$

In Equations (2)-(5), $B(0)$ and $D(0)$ are the direct and diffuse components of the horizontal radiation; $B_{0}$ is the solar constant; $\varepsilon_{0}$ is the eccentricity correction factor, $\beta$ is the inclination of the surface from the horizontal plane and $\theta_{\mathrm{ZS}}$ is the zenith angle.

Combining both models, the incidence irradiance on a tilted and dirty surface, $G_{\text {eff }}\left(\theta_{S}\right)$, can be modeled according to the global and diffuse irradiance on a clean and horizontal surface, $G(0)$ and $D(0)$ :

$$
\begin{aligned}
G_{\text {eff }}\left(\theta_{S}\right)= & \frac{T_{\text {soiled }}}{T_{\text {clean }}} \\
& \times\left[\left(1-\mathrm{FT}_{B}\right)\left(B+D_{C}\right)+\left(1-\mathrm{FT}_{D}\right) D_{I}+\left(1-\mathrm{FT}_{R}\right) R\right]
\end{aligned}
$$

As mentioned above, field measurements of horizontal radiation, $G(0)$ and $D(0)$, measured by both clean horizontal pyranometers, and irradiance on a tilted and dirty surface, $G_{\text {eff }}\left(\theta_{S}\right)$, measured by the reference unclean cells,

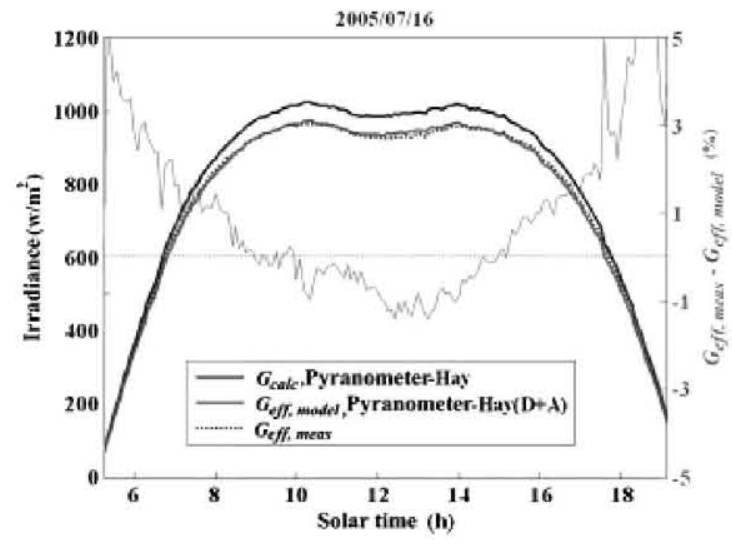

Figure 6. Measured, $G_{\text {eff, meast }}$ and calculated, $G_{\text {Calc, }}$, irradiance on the tracking surface during the date 16 July 2005. Calculated irradiance including dirt and angle of incidence effects $(D+A)$,

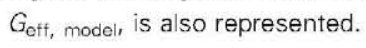

are available. The daily normal incidence transmittance, $T_{\text {soiled }} / T_{\text {clean }}$, and the optical energy losses on a tilted surface are calculated by the best fit of Equation (6) for each day of the period under analysis. Figure 6 presents the results of a certain day assuming that dirt remained constant throughout the day. Parameter $a_{r}$ was equal to 0.22 . The corresponding normal incidence transmittance was 0.96 . Errors are frankly small. The optical energy losses on a tilted surface resulted to be $0.5 \%$.

Similarly, the normal incidence transmittance and the optical energy losses can be calculated for a horizontal surface. Adapting Equation (6), the incidence irradiance on a horizontal and dirty surface, $G_{\text {eff }}\left(0, \theta_{S}\right)$, can be calculated according to

$$
G_{\text {eff }}\left(0, \theta_{S}\right)=\frac{T_{\text {soiled }}}{T_{\text {clean }}}\left[\left(1-\mathrm{FT}_{B}\right) B(0)+\left(1-\mathrm{FT}_{D}\right) D(0)\right]
$$

Figure 7 represents the best fit of Equation (7) to the empirical data recorded on the same date as in Figure 6, assuming the dirt on the surface was the same throughout the day. Parameter $a_{r}$ equaled 0.26 . The corresponding normal incidence transmittance was 0.92 . The optical energy losses on the horizontal surface were $12.1 \%$.

It is possible to refine the calculation considering the particular spectral responses of the pyranometers and solar cells. Some authors, such as Martín and Ruiz [13] or King [14] have suggested equations that allow that enhancement. The difference is noticeable mainly on the first and last moments of the day, when the air mass is the biggest. Figure 8 presents the result of correcting the measured irradiance of the calibrated cells by the equation proposed by D. L. King on the same date shown in Figures 6 and 7 . The values of $a_{r}$ and the normal incidence transmittance remain the same, whereas the optical energy losses on the tilted surface become $4.5 \%$. The optical energy losses on the horizontal surface become $11.4 \%$. Thus, to use or not to 


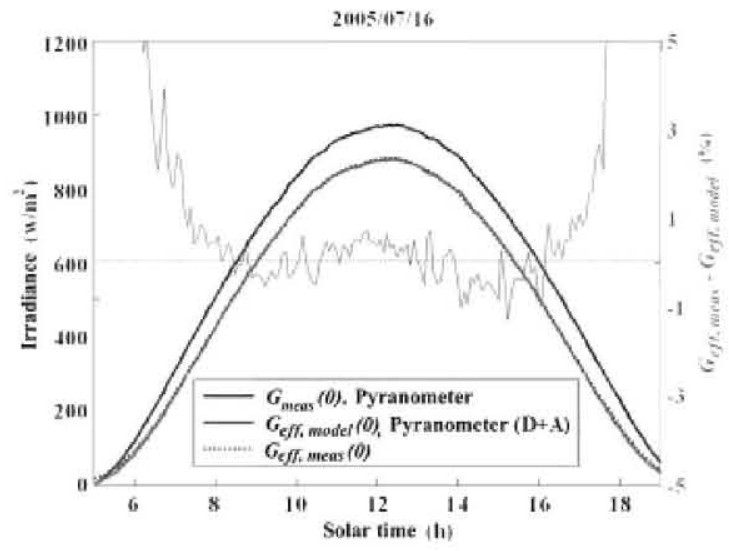

Figure 7. Horizontal irradiance measured by the references cells, $G_{\text {eff, meas }}(0)$, and the pyranometer, $G_{\text {meas }}(0)$, during the date 16 July 2005. Calculated horizontal irradiance including dirt and angle of incidence effects $(D \mid A), G_{\text {eff, model }}(0)$, is also represented.
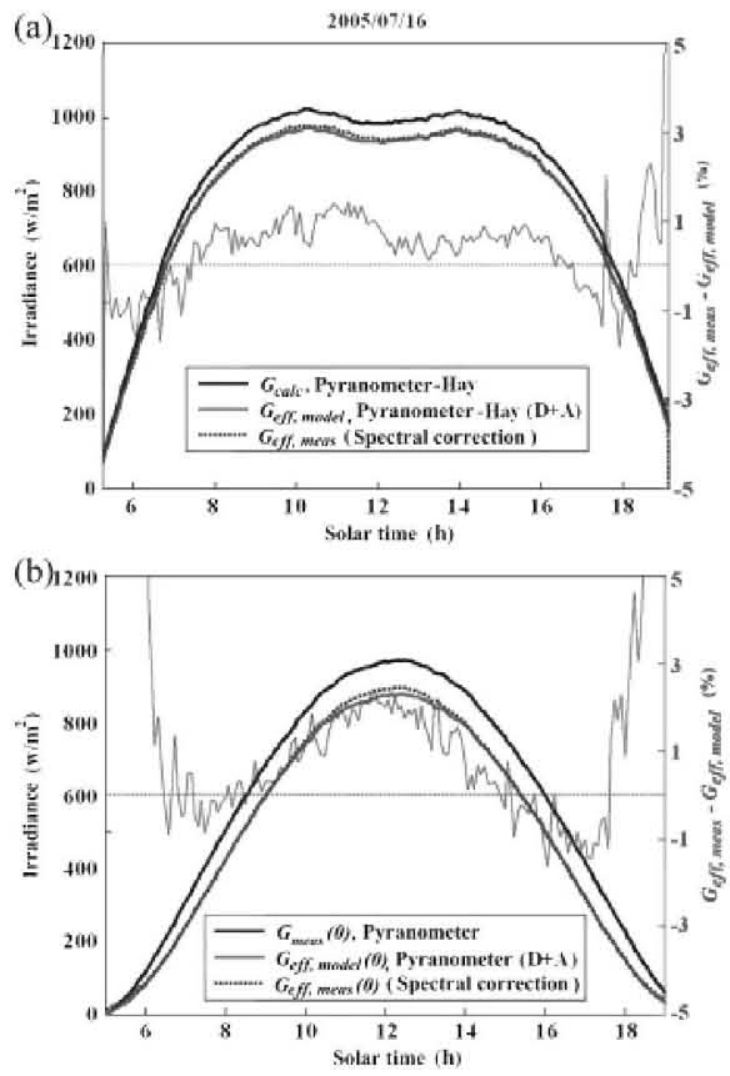

Figure 8. Measured (applying spectral correction) and calculated irradiance at the tilted surface (a), $G_{\text {eff, meas }}$ and $G_{\text {calc }}$ and at the horizontal plane (b), $G_{\text {eff, meas }}(0)$ and $G_{\text {meas }}(0)$, during the date 16 July 2005. Calculated irradiance including dirt and angle of incidence effects, $G_{\text {eff, model }}$ (tilted surface) and $G_{\text {eff, }}$ model $(0)$ (horizontal plane), is also represented. use a spectral correction involves, on a certain day, a difference of about $0.5 \%$ on the calculation of the optical energy losses. For other days analyzed, the difference was less.

\section{EXPERIMENTAL RESULTS}

Daily optical energy losses from February 2005 to May 2006 have been calculated using the method priory described. Figure 9 shows the energy losses corresponding to the clearest days. The bars represent daily rainfall during those months.

Daily optical energy losses vary, along the year, from 1 to $8 \%$ in the case of tracking surfaces, and from 8 to $22 \%$ in the case of horizontal surfaces. Clearly, rain contributes to clean the modules as long as the daily rainfall surpasses a certain value (about 4-5 $\mathrm{mm}$ ). The highest optical energy losses are registered during the late winter months, when coincidentally the rainfall is the lowest and least intense. During the summer months the optical energy losses were lower, although it is observable how the losses augment as dry days go by. This energy loss increase is about $0.1-0.2 \%$ per day. During this time of the year, storms contribute to maintain the modules acceptably clean. Additionally, Figure 9 shows significant difference between the energy losses of tracking surfaces and horizontal ones. This difference is more clearly depicted in Figure 10 which shows both the monthly energy losses at normal incidence and the additional monthly energy losses due to the AOI. The annual optical energy losses are represented by a horizontal black line.

The monthly soiling optical losses range from 2 to $6 \%$ on tracking surfaces, while in the case of horizontal surfaces the losses are higher but more uniform along the year (ranging from 7 to $9 \%$ ). The effect of the AOI on the horizontal surface is clearly observed, especially during the winter months. As a matter of fact, the optical losses due to the AOI are almost constant during the year, about $1 \%$ on tracking surfaces, whereas on horizontal surfaces they range from 2 to $3 \%$ during summer months to up to $8 \%$

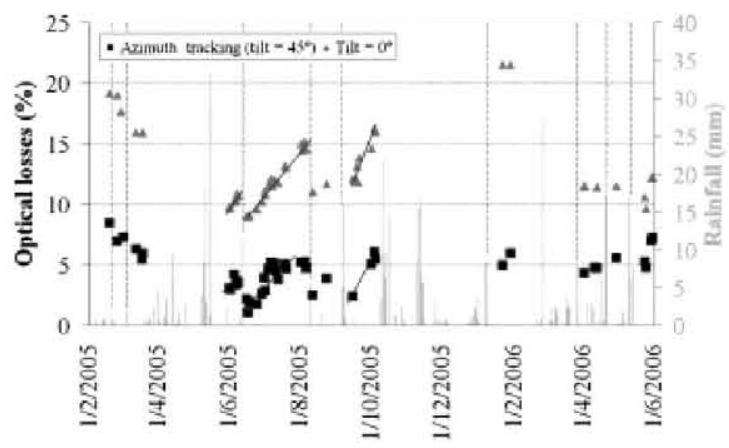

Figure 9. Optical losses in clear days from February 2005 to May 2006. Daily rainfalls during that period are included. 
(a)

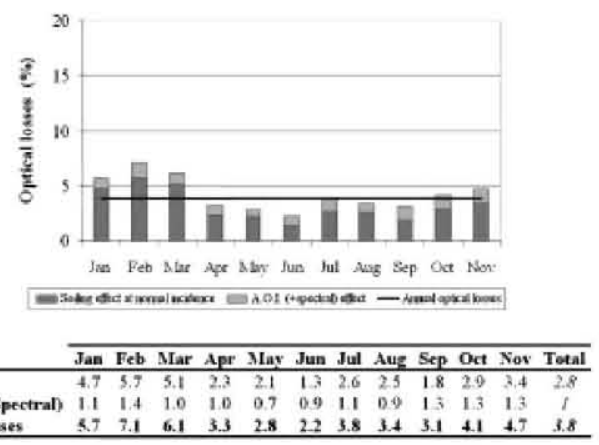

(b)

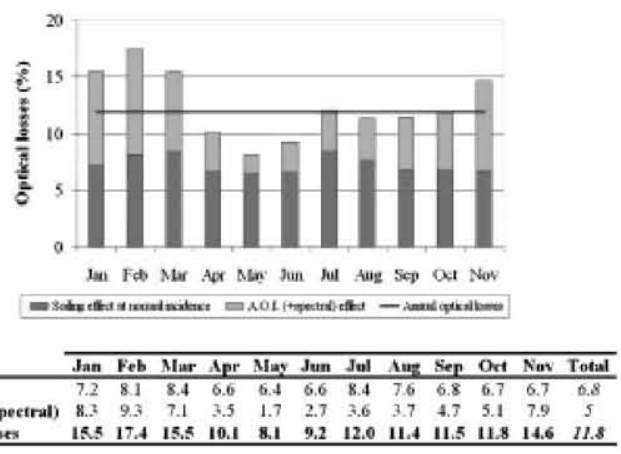

Figure 10. Monthly and annual optical losses (Soiling + A.O.I.) in 2005: (a) $45^{\circ}$ tilted surface, with azimuth tracking and (b) horizontal surface.

during certain winter months. In total, the annual optical energy losses on tracking surfaces and horizontal surfaces are 3.8 and $11.9 \%$, respectively.

\section{CONCLUSIONS}

This paper compiles field measurements of soiling and AOI annual energy losses measured at a PV plant located in northern Spain. These energy losses have been measured in azimuth trackers with $45^{\circ}$ tilted modules and also in fixed horizontal modules.

Along the period under study, daily optical energy losses ranged from 1 to $8 \%$ in the case of tracking surfaces and from 8 to $22 \%$ in the case of fixed horizontal surfaces. During dry periods, the losses increased about $0.1-0.2 \%$ per day. It has been confirmed that daily rainfalls above $4-5 \mathrm{~mm}$ noticeably clean the modules.

The energy losses due to dirt are notably higher on horizontal surfaces and also more uniform along the year. These losses ranged from 2 to $6 \%$ on the tracking surfaces and from 7 to $9 \%$ on the horizontal surfaces; showing that the cleaning effect of rainfall on horizontal surfaces is not as effective as it is on inclined ones. Additionally, the effect of the $\mathrm{AOI}$ is also more noticeable on horizontal surfaces, especially during winter months. The AOI losses were about $1 \%$ on tracking surfaces, whereas for horizontal surfaces the losses ranged from 2 to $3 \%$ during summer months to up to $8 \%$ during certain winter months.

It is worth mentioning that the cosine response of the pyranometer, for very high incidence angles $\left(>70^{\circ}\right)$, is not perfect. This could influence the AOI energy loss estimations. However, during the period under analysis, the radiation received upon a horizontal surface with $\mathrm{AOI}$ over $70^{\circ}$ is less than $7 \%$. If an error due to the pyranometer cosine response for these $\mathrm{AOI}$ of about $5 \%$ is considered, the energy loss total error would be less than $0.35 \%$. The error would be even less for tracking surfaces.

In summary, the annual optical losses of the plant were $3.8 \%$ (approximately, $1 \%$ of which were due to the AOI and the rest were due to dirt) in the case of tracking surfaces; and $11.9 \%$ in the case of horizontal surfaces (approximately, 5\% of which were AOI energy losses and the rest soiling energy losses).

The optical energy losses presented correspond to a rural area surrounded mainly by dry crops and forestry areas. The accumulated annual rainfall is about $370 \mathrm{~mm}$ and it is mainly concentrated in the spring and autumn months. There are scarce dust sources nearby the location (except for some plow cereal crops). Other dirtier locations (urban polluted areas or areas near quarries) might undergo higher losses.

\section{ACKNOWLEDGEMENTS}

The authors acknowledge and thank Dr José María Ruiz Pérez for his valuable comments.

\section{REFERENCES}

1. Duffie JA, Beckman WA. Solar Energy and Thermal Processes, 2nd edn. Wiley: New York, 1991.

2. Sjerps-Koomen EA, Alsema EA, Turkenburg WC. A simple model for PV reflection losses under field conditions. Solar Energy 1996; 57(6): 421-432.

3. Martin N, Ruiz JM. A new model for PV modules angular losses under field conditions. International Journal of Solar Energy 2002; 22(1): 19-31.

4. Martin N, Ruiz JM. Annual angular reflection losses in PV modules. Progress in Photovoltaics: Research and Applications 2005; 13: 75-84.

5. Hammond R, Srinivasan D, Harris A, Whitfield K, Wohlgemuth J. Effects of soiling on PV module and radiometer performance. Proceedings of the 26th PVSC, 1997; 1121-1124.

6. Becker H, Vaaben W, Herrmann W. Reduced output of solar generators due to pollution. Proceedings of the 14th European PV Solar Energy Conference and Exhibition, Barcelona, Spain, 1996.

7. Haeberlin H, Graf JD. Gradual reduction of PV generator yield due to pollution. Proceedings of the 2nd World Conference on Photovoltaic Solar Energy Conversion, Vienna, Austria, 1998. 
8. Kimber A, Mitchell L, Nogradi S, Wenger H. The effect of soiling on large grid-connected photovoltaic systems in California and the southwest region of the United States. Conference Record of the 2006 IEEE 4th World Conference on Photovoltaic Energy Conversion, Vol. 2; 2391-2395.

9. Vivar M, Herrero R, Martínez-Moreno F, Antón I, Sala G. Effect of soiling in PV concentrators: mechanisms of light dispersion and real field performance of soiled flat modules and CPV's. 23rd European Photovoltaic Solar Energy Conference and Exhibition, Valencia, Spain, September 2008.

10. Kimber A. The effect of soiling on photovoltaic systems located in arid climates. Proceedings of the 22nd European Photovoltaic Solar Energy Conference, Milan, Italy, 2007.
11. Michalsky JJ, Harrison LC, Berkheiser WE. Cosine response characteristics of some radiometric and photometric sensors. Solar Energy 54(6): 397-402. 1995.

12. Hay J, Mckay D. Estimating solar irradiance on inclined surfaces: a review and assessment of methodologies. International Journal of Solar Energy 3: 203240.1985.

13. Martin N, Ruiz JM. A new method for the spectral characterisation of PV modules. Progress in photovoltaics: research and applications $1999 ; 7(4)$ : 299310

14. King DL, Kratochvil J, Boison A, Bower W WE. Field experience with a new performance characterization procedure for photovoltaic arrays. 2nd World Conference and Exhibition on Photovoltaic Solar Energy Conversion, 1947-1952, Vienna, 1998. 